\title{
Improving PWR core simulations by Monte Carlo uncertainty analysis and Bayesian inference
}

\author{
E. Castro, C. Ahnert, O. Buss, N. García-Herranz, A. Hoefer, D. Porsch
}

\begin{abstract}
The Monte Carlo based Bayesian inference model MOCABA is applied to the prediction of reactor operation parameters of a PWR nuclear power plant. In this non perturbative framework, high dimensional covariance information describing the uncertainty of microscopic nuclear data is combined with measured reactor operation data in order to provide statistically sound, well founded uncertainty estimates of integral parameters, such as the boron letdown curve and the burnup dependent reactor power distribution. The performance of this methodology is assessed in a blind test approach, where we use measurements of a given reactor cycle to improve the prediction of the subsequent cycle. As it turns out, the resulting improvement of the prediction quality is impressive. In particular, the prediction uncertainty of the boron letdown curve, which is of utmost importance for the planning of the reactor cycle length, can be reduced by one order of rnagnitude by including the boron concentration measurement information of the previous cycle in the analysis. Additionally, we present first results of non-perturbative nuclear data updating and show that predictions obtained with the updated libraries are consistent with those induced by Bayesian inference applied directly to the integral observables.
\end{abstract}

\section{Introduction}

Best estimate plus uncertainty methodologies allow for a reli able quantification of safety margins for nuclear power plant operation and for the manufacturing, handling, storage and transport of nuclear fuel (Antila et al., 2003; D'Auria et al., 2008; lvanov et al., 2013; lvanova et al., 2013). This opens up the possibility to elimi nate unnecessary conservatism, which leads to improved plant performance through optimized core design and greater opera tional flexibility and to reduced fuel management costs. However, the evaluation of statistical confidence bounds for nuclear safety parameters requires a consistent statistical framework to combine and propagate uncertainties in nuclear data, technological data, and operational data.

Two different approaches are currently used to propagate nuclear data uncertainties to integral observable uncertainties: perturbation theory and Monte Cario sampling.

The perturbation theory approach has been successfully used for more than a half a century (Uchasev et al., 1964; Gandini, 1967) and has been implemented in many different computer

codes. Within this framework, integral functions of nuclear data are approximated by their first arder series expansions, which implies that the integral observable uncertainties are expressed as linear transformations of nuclear data covariances defined by the sensitivities of the integral observables to the nuclear data; see e.g. (Broadhead et al., 2004). Typically, the applied nuclear transport codes have to be upgraded for computing the sensitivity coefficients. As a first arder approximation, this approach yields sufficiently accurate uncertainty estimates only under the condi tion that the relevant nuclear data uncertainties are not too large.

In recent years, the Monte Cario sampling approach has been playing an increasingly important role in the uncertainty propaga tion of nuclear data to integral observables (Koning and Rochman, 2008; Sanz et al., 2008; Zwermann et al., 2010; Buss et al., 2011; Williams et al., 2012; Wieselquist et al., 2013). This method con sists in random sampling of nuclear data parameters optionally together with technological and operational parameters from their joint uncertainty distribution, where each random sample is used in a different computation of the integral observable of 
interest. Finally, uncertainty estimations for the integral observ ables are obtained from the statistics of Monte Carlo computations. Since this approach does not rely on perturbation theory, any inac curacies due to omitting higher order effects can be ruled out a pri ori. Additionally, the transport codes can be used as so called black boxes and do not need to be adapted for computing sensitivity coefficients, typically by implementing adjoint flux computation capabilities.

A strong point of first order perturbation theory is that it can be combined with the Generalized Linear Least Squares (GLLS) method (Cecchini et al., 1965; Humi et al., 1965; Hemment et al., 1966; Broadhead et al., 2004; De Saint Jean, 2010; Salvatores et al., 2013) to include measurements of integral observables in order to improve the knowledge about the nuclear data and, con sequently, about the integral observables depending on them.

The Monte Carlo approach, in contrast, has in the past been lim ited by the fact that an equally rigorous framework as the GLLS method was missing. However, this limitation has been removed recently by the development of the MOCABA methodology (Hoefer et al., 2015). As for the GLLS method, MOCABA is based on a Bayesian model that permits the inclusion of information from integral measurements to improve the prediction of integral observables. A major advantage of this approach is that MOCABA updating can be applied directly to the integral observables with out taking the detour via nuclear data updating. On the other hand, since nuclear data can be seen as just a special case of an integral observable, MOCABA can also be used for non perturbative nuclear data updating, which may be an attractive alternative to the GLLS approach for the generation of adjusted nuclear data libraries (Salvatores et al., 2014). Mathematically, MOCABA can be seen as a non perturbative generalization of the GLLS framework, which is shown by the fact that applying first order perturbation theory to the MOCABA model yields the well known GLLS formulas (Hoefer et al., 2015).

In this work, the performance of Monte Carlo based Bayesian inference is tested for application in reactor physics. The question we address is what we can learn from previous reactor measure ments for the prediction of future reactor cycles. As a test case, we consider two consecutive burnup cycles of a Spanish PWR nuclear power plant, denoted as Cycle A and Cycle B, where we attempt to predict Cycle B based on measurements of Cycle A. Here we focus on two reactor observables: the boron letdown curve, representing the critical boron concentration in the reactor as a function of burnup, and the reactor power distribution defined by the power values of the individual fuel assemblies in the reactor core. For the propagation of nuclear data uncertainties to the con sidered reactor observables, random samples of ENDF/B VII.1 nuclear data files (Chadwick et al., 2011) are generated with the aid of the NUDUNA Monte Carlo code (Buss et al., 2011), which are then converted to random libraries for core simulations with the extensively validated SEANAP PWR analysis system (Ahnert and Aragonés, 1985; Ahnert et al., 1999). The prior uncertainty dis tributions of the boron concentration and the assembly wise power distribution are obtained from a statistical evaluation of the SEANAP results calculated with the different random libraries. Subsequently, the MOCABA updating procedure is used to improve the prior predictions of the reactor observables of Cycle $B$ by including measurements of Cycle A. The predictive power of this approach is verified by comparing the predicted reactor observ ables of Cycle B to the actual measured values. Finally, we compare the predictions from direct integral observable updating to those obtained via MOCABA updating of the SEANAP nuclear data library.

The main objective of our analyses is to verify the usefulness of the MOCABA framework applied to full reactor core simulations from the point of view of its ability to improve reactor cycle predictions.
The paper is structured as follows. In Section 2, we give a brief introduction to the SEANAP core analysis system, the NUDUNA nuclear data Monte Carlo code and the MOCABA updating scheme, and we describe how they are combined in our reactor uncertainty analysis. In Section 3 we apply this methodology to the PWR test case described above. After a discussion of the results, we end with conclusions and outlook.

\section{Description of the NUDUNA/ MOCABA methodology applied to SEANAP core analysis}

The procedure we use for reactor cycle prediction is divided in three steps:

- Generation of random nuclear data libraries with the aid of the NUDUNA Monte Carlo code,

- Performing SEANAP burnup simulations for Cycles A and B for each of the random library inputs,

- MOCABA updating of the prediction of Cycle B using measure ments of Cycle $A$.

In the following, the codes involved in this procedure are described, together with the applied methodology.

\subsection{SEANAP: PWR core analysis}

SEANAP is a system for the simulation and analysis of PWR cores, developed at Universidad Politécnica de Madrid (Ahnert and Aragonés, 1985; Ahnert et al., 1999). It consists of a chain of concatenated codes covering the different tasks needed to perform PWR core simulations, and it has been used for the simulation of many cycles of Spanish PWRs for the last 25 years, with good agreement between the simulated and the measured values. This system can calculate a wide variety of parameters: boron concen tration, peak factors, axial offset, reactivity coefficients, power per fuel assembly, among many others. The deviations between the simulated and measured values are analyzed in Ahnert et al. (1988) and are below 20 ppm for the boron concentration and below $5 \%$ for the power distribution. The SEANAP system is a fast simulation tool that allows the computation of many different observables at the core level. Hence, it is well suited to analyze the impact of uncertainties in the basic nuclear data on global core parameters.

The main components of this system are presented in Fig. 1. The MARIA subsystem consists of the PREWIM, WIMS D4 and POSWIM codes. The PREWIM code generates the input files required by WIMS D4 for all the fuel assemblies, covering the parameter space of the local physical variables, using a cylindricalized model of the square PWR assembly, which provides an efficient treatment of the PWR fuel assembly. The WIMS D4 lattice code (Ahnert, 1980; Halsall, 1980) calculates the PWR fuel assembly in the annular cluster geometry by $S_{N}$ neutron transport calculation using a 69 energy group nuclear data library. As a result, the cross sections for each fuel assembly are obtained, homogenized at the assembly level and at the pin level.

COBAYA 2 is a 2D two group pin by pin diffusion code, with a fine mesh finite difference method. Its purpose is to compute nodal discontinuity factors and hot pin to node average power ratios, at some reference conditions, that are used in the SIMULA core simulator. SIMULA is a 3D nodal code, using four nodes per fuel assembly, an axial mesh of 34 nodes and simplified closed channel thermal hydraulics. The solver is a linear discontinuous finite difference scheme for synthetic coarse mesh few group dif fusion calculation (Aragonés and Ahnert, 1986). It uses the nodal 


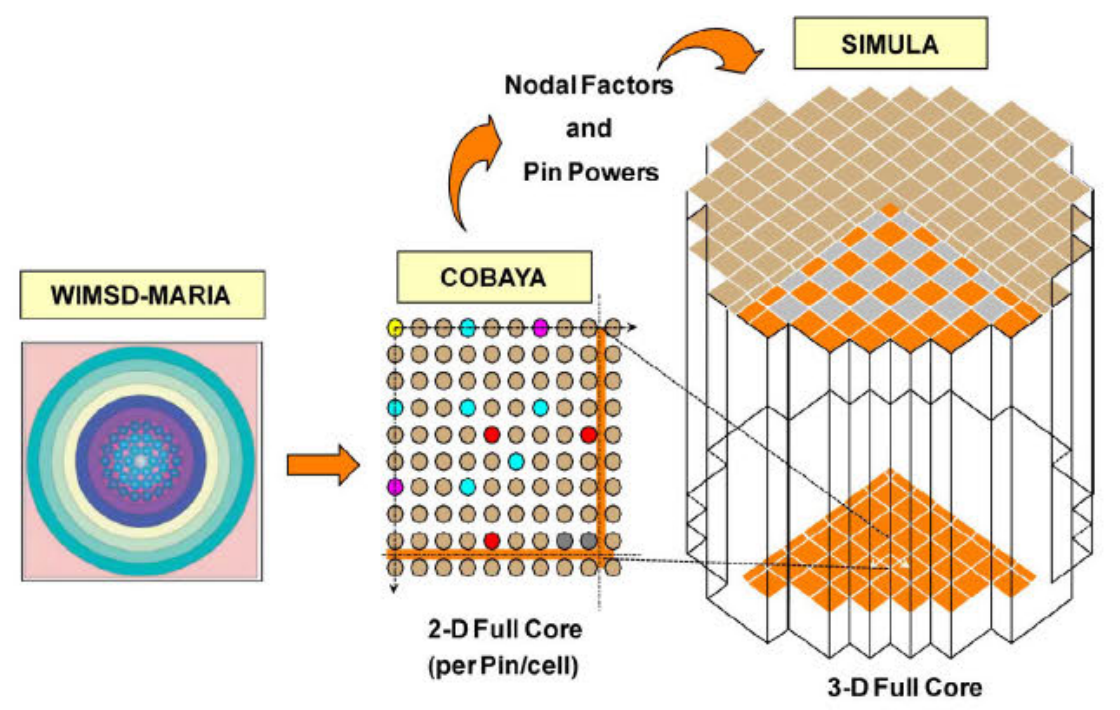

Fig. 1. Scheme of the PWR core analysis system SEANAP.

discontinuity factors provided by COBAYA 2 for each node as a function of the burnup.

\subsection{NUDUNA: nuclear data random sampling}

The NUDUNA (NUclear Data UNcertainty Analysis) program, developed by AREVA GmbH (Buss et al., 2011), provides Monte Carlo sampling of nuclear data, generating random libraries that can be used in core simulations.

This code reads evaluated nuclear data files in ENDF 6 format (CSEWG, 2010), and provides random libraries based on the covari ance information included in the evaluations for the following data:

- average fission neutron multiplicities (File 1)

- resonance parameters (File 2)

- cross sections (File 3)

- angular distributions (File 4)

- decay data (File 8, Section 457)

NUDUNA is coupled to the NJOY nuclear data processing system (MacFarlane et al., 2012) in order to automatically generate nuclear data inputs for different transport codes. For this work, the processing of the files to the WIMSD format has been imple mented using the WIMSR module of NJOY, based on (Leszczynski et al., 2007 and MacFarlane and Kahler, 2010). NUDUNA also sup ports the ACE format of MCNP (X 5 Monte Carlo Team, 2003), the AMPX format of SCALE (Oak Ridge National Laboratory, 2009), and the APOLLO II neutron library format (Sanchez et al., 1988).

Here we apply WIMSD formatted NUDUNA samples in SEANAP transport calculations. The resulting statistics of reactor operation parameters reflects their uncertainty due to nuclear data uncertainties.

\subsection{MOCABA: Bayesian inference of predictions}

MOCABA is a mathematical framework developed by AREVA $\mathrm{GmbH}$ to combine Monte Carlo sampling and Bayesian inference in order to achieve improved predictions of integral functions of nuclear data (Hoefer et al., 2015). In this framework, the vector of application case observables $\mathbf{y}_{A}$, i.e. the parameters we want to predict, and the vector of benchmark observables $\mathbf{y}_{B}$, for which measurements are available, are collected in a combined vector y $\quad\left(\mathbf{y}_{A}^{T}, \mathbf{y}_{B}^{T}\right)^{T}$.

The fact that the application case and benchmark observables are known with limited precision is taken into account by treating $\mathbf{y}$ as a random vector, and the prior distribution $\mathrm{p}(\mathbf{y})$, reflecting the uncertainty of $\mathbf{y}$ due to uncertainties of nuclear data, technological parameters and operational parameters, is assessed through Monte Carlo sampling of these parameters and subsequent computation of $\mathbf{y}$ for each random sample. Then $\mathrm{p}(\mathbf{y})$ is estimated from the resulting statistics of $\mathbf{y}$ computations. For a normal distribution model, this means to estimate the prior mean vector $\mathbf{y}_{0}$ and the prior covariance matrix $\Sigma_{0}$ from the Monte Carlo data (Hoefer et al., 2015) ${ }^{2}$ :

$\mathbf{y}_{0} \quad\left(\mathbf{y}_{0 A}^{T}, \mathbf{y}_{0 B}^{T}\right)^{T}, \quad \mathbf{\Sigma}_{0} \quad\left(\begin{array}{ll}\mathbf{\Sigma}_{0 A} & \mathbf{\Sigma}_{0 A B} \\ \mathbf{\Sigma}_{0 A B}^{T} & \mathbf{\Sigma}_{0 B}\end{array}\right)$.

Measurements of the benchmark vector $\mathbf{y}_{B}$ and/or linear con straints on $\mathbf{y}$, represented by a vector $\mathbf{v}$, are expressed in terms of a likelihood function $\mathrm{p}(\mathbf{v} \mid \mathbf{y})$, which depends on the distribution used. To add this information to the prior information, Bayes' the orem is applied, which yields the posterior distribution:

$\mathrm{p}(\mathbf{y} \mid \mathbf{v}) \propto \mathrm{p}(\mathbf{v} \mid \mathbf{y}) \mathrm{p}(\mathbf{y})$.

For a normal distribution model, the Bayesian inference replaces the prior mean vector $\mathbf{y}_{0}$ and the prior covariance matrix $\Sigma_{0}$ by the posterior mean vector $\mathbf{y}^{*}$ and the posterior covariance matrix $\Sigma^{*}$ :

$$
\mathbf{y}^{*} \quad\left(\mathbf{y}_{A}^{* T}, \mathbf{y}_{B}^{* T}\right)^{T}, \quad \mathbf{\Sigma}^{*} \quad\left(\begin{array}{cc}
\Sigma_{A}^{*} & \Sigma_{A B}^{*} \\
\Sigma_{A B}^{* T} & \Sigma_{B}^{*}
\end{array}\right) .
$$

The impact of the benchmark measurements on the prediction of the application case observables is determined by the matrix $\Sigma_{0 A B}$ of prior covariances between application case and benchmark observables. If the corresponding correlations are close to 1 , mea surements of $\mathbf{y}_{B}$ have the potential to significantly improve the prediction of $\mathbf{y}_{A}$. This is generally the case if application case and benchmarks are similar, in the sense that they show similar responses to variations in the input parameters, in particular nuclear data. Due to its general formulation, the MOCABA frame work can be applied to the prediction of any integral observable,

\footnotetext{
${ }^{2}$ A more general class of distribution models can be accessed by making use of invertible variable transformations (Hoefer et al., 2015).
} 
and any integral measurement can be used to update the predic tions. As mentioned above, MOCABA can also be used for non perturbative updating of nuclear data libraries. For a more detailed description see (Hoefer et al., 2015).

\subsection{Methodology of reactor cycle prediction}

In the following, SEANAP, NUDUNA and MOCABA are used in combination to improve the predictions of reactor operation parameters in a given burnup cycle of a PWR (Cycle B) based on measurements and simulations of the previous cycle (Cycle A).

Fig. 2 shows a scheme of the applied methodology, which is divided in three steps. First, a sufficient number (we use $200^{3}$ ) of random nuclear data libraries are generated with NUDUNA, using covariance data from the ENDF/B VII. $1^{4}$ evaluation (Chadwick et al., 2011) for the most important isotopes (Díez et al., 2015), being ${ }^{235} \mathrm{U},{ }^{238} \mathrm{U},{ }^{239} \mathrm{Pu},{ }^{1} \mathrm{H},{ }^{16} \mathrm{O}$ and ${ }^{10} \mathrm{~B}$. These random samples are com bined with the reference library of WIMS using the WILLIE code (Trkov, 2008), yielding a set of random nuclear data libraries to be used in core simulations with SEANAP, which is the second step. The core simulation results are then used in a statistical analysis to estimate the prior mean values and covariances of the Cycle A and Cycle B observables.

In the last step, measurement information of Cycle $A$ is added by applying MOCABA updating to the prior Cycle $A$ and Cycle B pre dictions. Hence, Cycle $A$ is used as a benchmark to improve the pre diction of Cycle B. ${ }^{5}$

Uncertainty contributions of system parameters (technological and operational parameters) are neglected in the procedure described above, even though they could be included in the MOCABA framework; see Section 2.3. Hence, the analysis pre sented in this paper is based on the assumption that the integral observable uncertainties are governed by nuclear data uncertain ties. This assumption may be motivated by the following consider ations. Since Cycle A and Cycle B are characterized by common components (same reactor, re used fuel assemblies, etc.), their observables are not independent with respect to uncertainties of the corresponding system parameters, and the related covariances between Cycle A and Cycle B observables lead to reduced uncer tainties of Cycle B predictions based on Cycle A measurements. This error cancelation reduce the impact of the system parameter uncertainties on the integral observable uncertainties, which leads us to the above assumption that system parameter uncertainties may be neglected compared to nuclear data uncertainties. In Sec tion 3 , the validity of this assumption will be tested by comparing Cycle B predictions to Cycle B measurements.

\section{Applications}

Here we consider two different reactor observables: the boron letdown curve, defining the time dependent critical boron concen tration in the reactor coolant during a burnup cycle, and the fuel assembly wise reactor power distribution. For the boron letdown curve, the components of the integral observable vectors are the boron concentrations, and for the reactor power distribu tion they are the power values of the individual fuel assemblies in the reactor core, at different times during the burnup cycle.

\footnotetext{
${ }^{3}$ It was verified that 200 samples are enough by splitting the set of random samples.

${ }^{4}$ Already in Ref. Diez et al. (2015) it was shown that covariances of the total and prompt multiplicities of U-235 are inconsistent. In this analysis, the prompt multiplicities of U-235 are varied according to their covariance data, and the total multiplicities are determined as sums of prompt and delayed multiplicities.

${ }^{5}$ Although the MOCABA framework allows linear constraints to be applied, for this application this feature is not used.
}

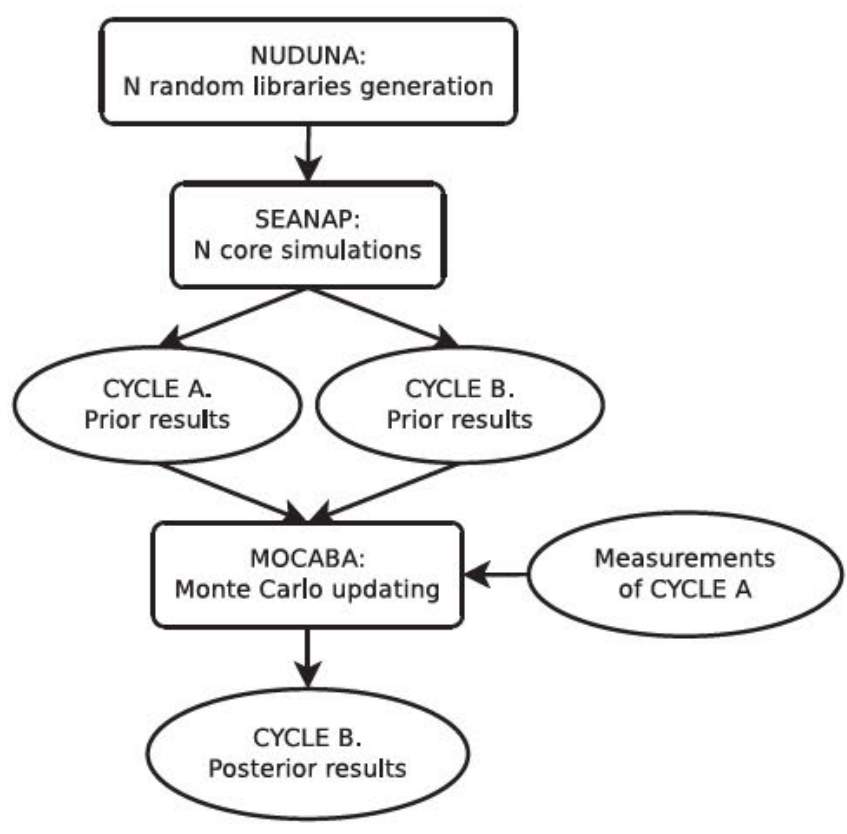

Fig. 2. Scheme of the methodology.

The analyzed reactor contains 157 fuel assemblies, with a rated thermal power of $2775 \mathrm{MWt}$, using fresh and previously used fuel, and WABA rods as burnable absorber. The refueling pattern for both cycles is quite similar, although the number of burnable absorber rods in the fuel assemblies is higher in Cycle A. The refu eling patterns for both cycles are presented in Fig. 3, where all fresh fuel assemblies have an initial enrichment of $3.6 \mathrm{w} / \mathrm{o}$, except for the fuel assemblies with 20 WABA rods in Cycle A, whose enrichment is $3.24 \mathrm{w} / \mathrm{o}$.

Measurements at 10 different burnup points of Cycle A are used to update the Cycle B predictions for 12 different burnup values. It is important to emphasize that only measurements of Cycle A are used in the updating procedure, and comparisons of Cycle B predic tions to Cycle B measurements are only made afterwards to assess the predictive power of the methodology. The uncertainty in the boron concentration measurements is $6 \mathrm{ppm},{ }^{6}$ that is $0.6 \%$ at the beginning of the cycle (BOC); and the uncertainty in the power mea surements is $5 \%$. These measurements are assumed to be indepen dent, that is, uncorrelated.

\subsection{Boron letdown curve of a burnup cycle}

The evolution of the critical boron concentration during a reac tor cycle is a measure of the cycle length. Hence, its prediction is essential for reactor operation planning. Fig. 4 shows the prior uncertainty in the predicted critical boron concentration of Cycle $B$ due to nuclear data, obtained by application of the NUDUNA ran dom libraries to the SEANAP calculations.

The total standard deviation due to all considered isotopes decreases slightly with burnup from a maximum value of $46 \mathrm{ppm}$ at BOC to a value of $40 \mathrm{ppm}$ at the end of the cycle (EOC).

${ }^{238} \mathrm{U}$ is the principal uncertainty contributor for burnup values up to $8 \mathrm{GWd} / \mathrm{t}$ heavy metal. Its contribution decreases continu ously from $36 \mathrm{ppm}$ at BOC to $25 \mathrm{ppm}$ at EOC. The uncertainties due to ${ }^{235} \mathrm{U}$ and ${ }^{239} \mathrm{Pu}$ have opposite behaviors. ${ }^{235} \mathrm{U}$ is consumed during the cycle, so its importance is decreasing, with an effect of $22 \mathrm{ppm}$ at BOC and $14 \mathrm{ppm}$ at EOC. On the other hand, ${ }^{239} \mathrm{Pu}$

\footnotetext{
${ }^{6}$ The boron concentration values presented here refer to mass ratios of natural boron in the reactor coolant.
} 

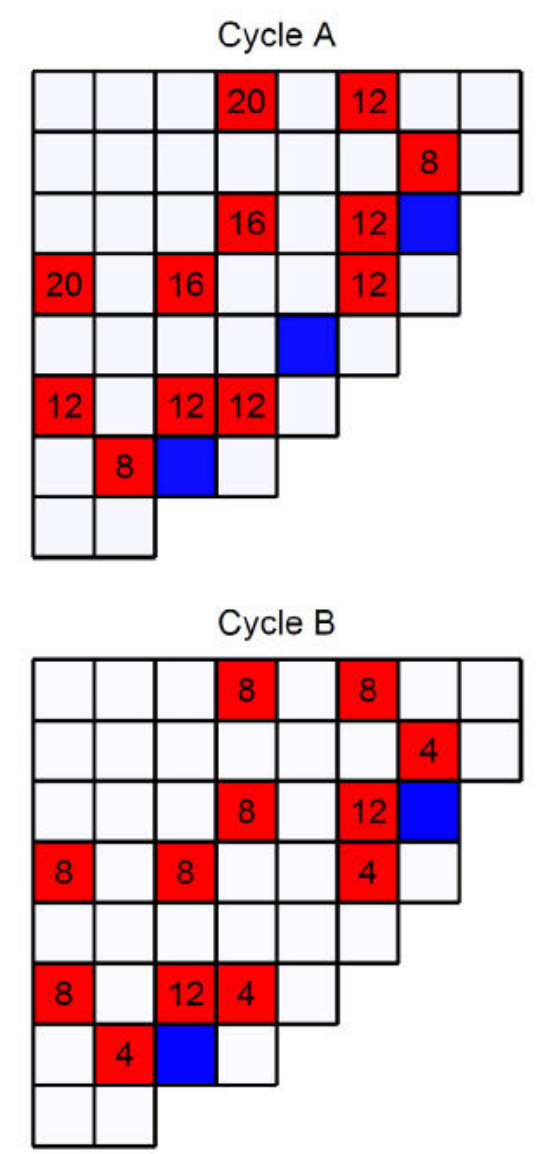

Fig. 3. Refueling pattern for the two cycles In blue: fresh fuel without burnable absorber. In red: fresh fuel with burnable absorber, the number indicates the number of rods with poison. In white: old fuel. (For interpretation of the references to colour in this figure caption, the reader is referred to the web version of this article.)

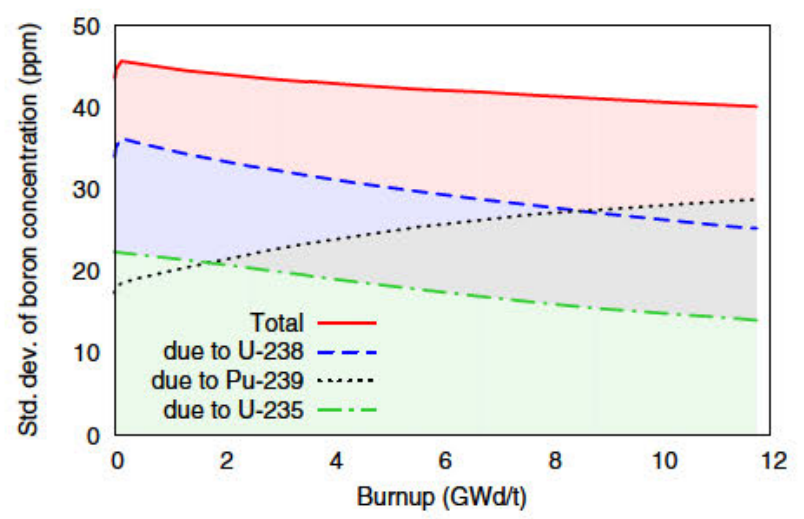

Fig. 4. Prior results for the standard deviation due to nuclear data uncertainties taken from ENDF/B-VII.1 of the boron concentration for Cycle B as a function of burnup.

is formed from ${ }^{238} \mathrm{U}$ during the cycle, so its uncertainty contribu tion increases from $19 \mathrm{ppm}$ at BOC to $29 \mathrm{ppm}$ at EOC.

The remaining materials have a much lower impact on the uncertainty of the boron concentration. Hydrogen and oxygen (in moderator and fuel) provide a basically constant uncertainty con tribution of approx. $1 \mathrm{ppm}$ and $5 \mathrm{ppm}$, respectively; ${ }^{10} \mathrm{~B}$ causes $1 \mathrm{ppm}$ uncertainty at BOC, and its impact is fully negligible at EOC.

A similar analysis of the prior uncertainties in the boron con centration was performed by Cabellos et al. (2014) with the Total
Monte Carlo (TMC) code (Koning and Rochman, 2008), which was based on the TENDL 2012 nuclear data evaluation (Koning and Rochman, 2011). Comparing the results derived by Cabellos et al. (2014) to the results presented above, shows that uncertain ties in the boron concentration are much larger for TENDL 2012/ TMC than for ENDF/B VII.1/NUDUNA, and also the relative impor tance of the isotopes differs ${ }^{7}$. This confirms that the choice of a nuclear data evaluation may have a large impact on predictions and their corresponding uncertainties, as has already been demon strated by Díez et al. (2015).

Finally, Bayesian inference is applied to the prior boron letdown curve with the MOCABA procedure, taking into account measure ments and simulations of the previous cycle, i.e. Cycle A. The cor responding outcomes are shown in Table 1 and Fig. 5. Two conclusions can be drawn from these results. First, for all burnup values, considering the previous cycle measurements leads to a better prediction of the boron letdown curve, i.e. the deviation between predicted and measured values is significantly reduced. Second, the uncertainty in the boron calculation is reduced by one order of magnitude, from values higher than $40 \mathrm{ppm}$ to values around $3 \mathrm{ppm}$, which means a huge improvement for the predic tion of a reactor cycle and, hence, for reactor operation planning.

The impressive improvement in the prediction of the boron let down curve by including measurements of the previous cycle is explained by Fig. 6. It shows large correlations between the boron values of Cycle A and Cycle B in the range 0.920 .99 , which reflects a high similarity between application case and benchmark, i.e. Cycle B and Cycle A.

Fig. 7 shows the convergence of the posterior boron concentra tion at a burnup of $7.72 \mathrm{GWd} / \mathrm{t}$ as a function of the number of benchmarks included in the Bayesian inference. The uncertainty is reduced with increasing number of benchmarks taken into account and thus the amount of information considered. Already including a single benchmark leads to a dramatic uncertainty reduction. Adding further measurements includes additional infor mation, which leads to even better predictions. However, the sim ulation results for the different burnup steps are correlated, and thus the amount of new information of an additional measurement is limited. Hence, the impact of the first measurement is the most prominent.

\subsection{Effects of nuclear data updating on boron letdown curve}

There is a different way of obtaining updated boron concentra tions using MOCABA, which is updating the WIMS nuclear data library using the measurements of Cycle A, and performing after that the SEANAP simulation for Cycle B with the updated library as input. For this purpose, the information of ${ }^{235} \mathrm{U},{ }^{238} \mathrm{U},{ }^{239} \mathrm{Pu}$ and ${ }^{1} \mathrm{H}$ has been updated in the 69 energy group WIMS reference library.

In order to update the nuclear data, the information of the dif ferent materials and energy groups is included in MOCABA, which performs the updating based on the simulations and measure ments of cycle A. In this case, vector $\mathbf{y}$ contains the sampled WIMS library values, and the corresponding integral observable simula tions of cycle A. After the execution of MOCABA, the outcome is used to construct an updated library in the WIMS format.

In Fig. 5 the results obtained with direct updating of the boron letdown curve are compared to results obtained with the updated nuclear data library. The results obtained with the two different

\footnotetext{
${ }^{7}$ In (Cabellos et al., 2014), the thermal scattering of the hydrogen in the water molecule is considered, while in this work only hydrogen cross sections are perturbed. This explains the differences for this material in the two studies, indicating that thermal scattering libraries might be an important source of uncertainty.
} 
Table 1

Boron concentrations for Cycle B as a function of burnup.

\begin{tabular}{cllllll}
\hline $\begin{array}{l}\text { Burnup } \\
(\mathrm{GWd} / \mathrm{t})\end{array}$ & $B C_{\text {prior }}$ & $\sigma_{\text {prior }}$ & $B C_{\text {posterior }}$ & $\sigma_{\text {posterior }}$ & $\sigma_{\text {priar }} / \sigma_{\text {posterior }}$ & $B_{\text {measured }}$ \\
\hline 0.13 & 986 & 46 & 986 & 4.2 & 11 & 983 \\
1.34 & 867 & 45 & 868 & 3.3 & 13 & 874 \\
2.49 & 763 & 44 & 767 & 2.7 & 16 & 771 \\
2.84 & 733 & 44 & 737 & 2.6 & 17 & 740 \\
3.59 & 664 & 43 & 668 & 2.4 & 18 & 672 \\
4.44 & 590 & 43 & 595 & 2.3 & 18 & 596 \\
5.55 & 487 & 42 & 494 & 2.2 & 19 & 496 \\
6.69 & 383 & 42 & 391 & 2.4 & 18 & 394 \\
7.72 & 290 & 42 & 298 & 2.6 & 16 & 402 \\
8.82 & 197 & 41 & 206 & 2.8 & 15 & 202 \\
10.28 & 74 & 41 & 85 & 3.2 & 13 & 87 \\
11.35 & -15 & 40 & -4 & 3.5 & 11 & 3 \\
\hline
\end{tabular}

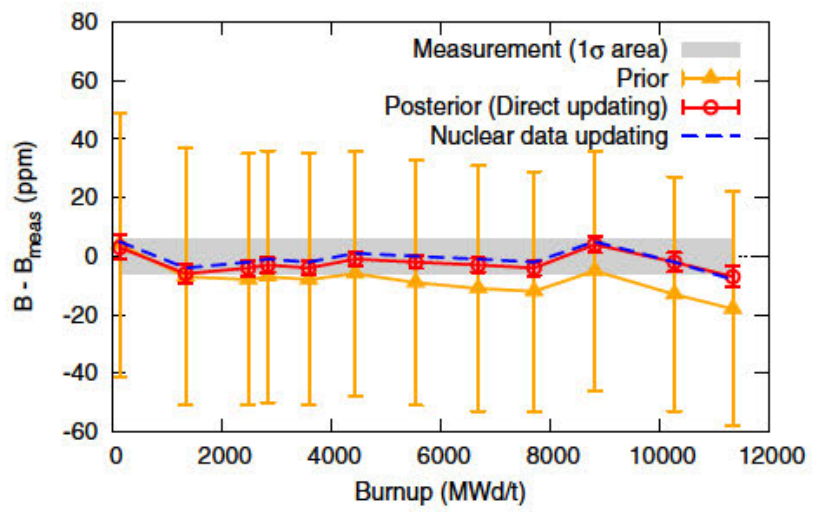

Fig. 5. Differences between measured boron concentrations and respective prior predictions, posterior predictions, and predictions obtained with a MOCABAupdated nuclear data library. The error bars represent the respective $1 \sigma$ uncertainties.

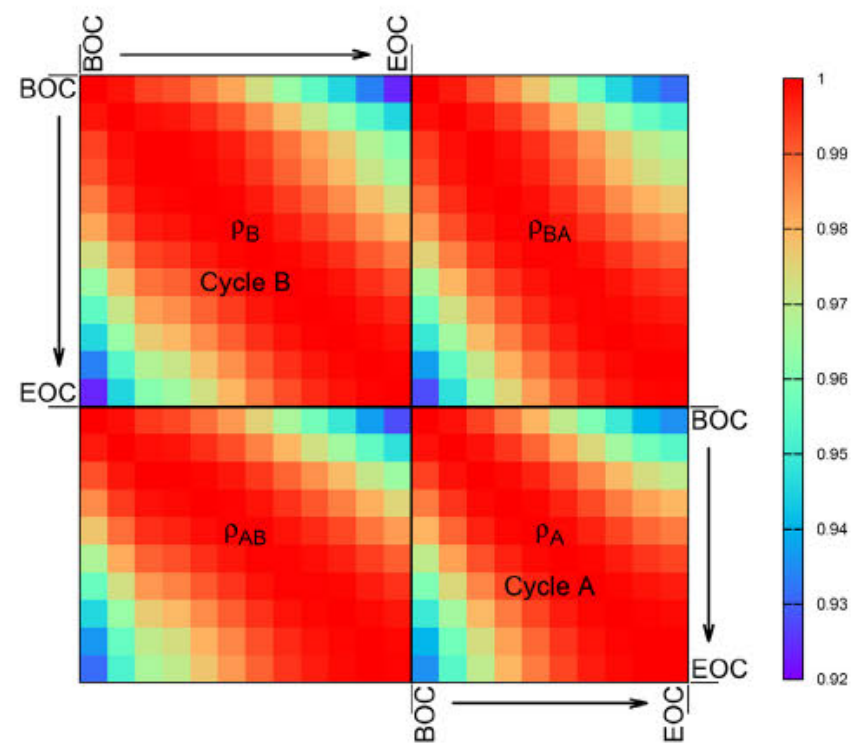

Fig. 6. Prior correlation matrix of boron concentration values for Cycle A and Cycle B at different burnup steps.

methods are consistent with each other, differing by less than $2 \mathrm{ppm}$.

Note that our study does not include other uncertainty contri butions than nuclear data uncertainties, e.g., no uncertainties related to technological parameters or to neutronics and thermal

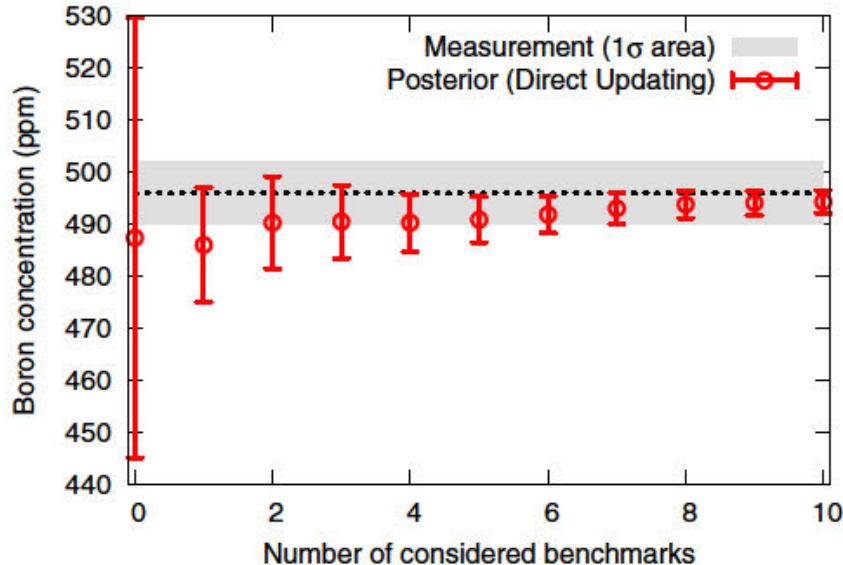

Fig. 7. Convergence of the MOCABA updating procedure for increasing numbers of considered benchmarks for a burnup of $7.72 \mathrm{GWd} / \mathrm{t}$

hydraulic models are taken into account. This ignorance could result in adapting nuclear data in order to balance deficiencies in the description of other input values, which might result in unphysical updates of the nuclear data. To check this effect, we compare in Fig. 8 the differences between original and updated library and normalize the difference to the standard deviation of the original library. The largest deviations can be observed for the ${ }^{239} \mathrm{Pu}$ fission cross sections, and the modifications are most prominent in the low energy groups. However, the differences are always smaller than the standard deviation of the original val ues and can be explained by its uncertainties. Nevertheless, the update could compensate other deficiencies, and thus it is to be checked in future studies whether the updated library obtained for one specific reactor also improves the description of other reactors.

\subsection{Power per fuel assembly in a burnup cycle}

Fig. 10 shows on the left hand side NUDUNA/ SEANAP uncer tainty estimates for the relative power per fuel assembly in Cycle $\mathrm{B}$ at BOC and EOC. Here, the relative power values are the respec tive fuel assembly wise power values divided by the mean power per fuel assembly in the reactor core. The uncertainty is largest in the center of the core and near the boundary, as has also been observed by Klein et al. (2012), where it is demonstrated that this effect is a consequence of the power normalization.

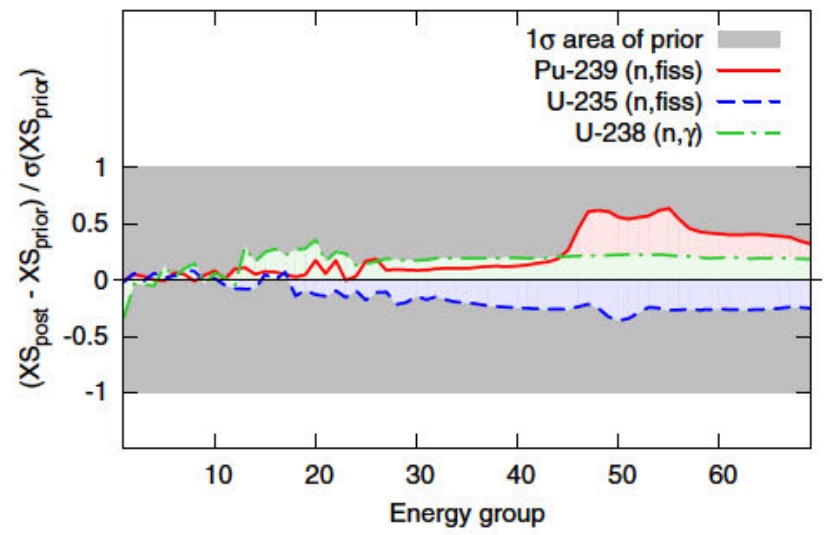

Fig. 8. Cross section updates normalized to one standard deviation. Fast energy groups are from 1 to 14 , resonant groups are from 15 to 27 , and thermal groups are from 28 to 69 . 
The relative powers per fuel assembly have been determined during Cycle A for each of the 47 fuel assembly positions in the considered quarter of the core and for 10 burnup steps, so there exist 470 power benchmark values. The right hand graphs of Fig. 10 show the Cycle B posterior uncertainties, which have been obtained with MOCABA based on the 470 Cycle A benchmarks. The maximum uncertainty reduction amounts to approximately $50 \%$. The average reduction depends on burnup (for $2842 \mathrm{MWd} / \mathrm{t}$ : $47 \%$, for $10284 \mathrm{MWd} / \mathrm{t}: 29 \%)$. This can be explained by the fact that Cycle A was shorter than Cycle B (by 0.5 GWd/t) and, consequently, the correlations between Cycle A and Cycle B EOC simulations turn out to be less prominent.

The correlations between the power values of the two different cycles are significantly smaller than those between the boron con centrations, where all correlations were over 0.92 . This is caused by differences of the cycles: burnable poison, fuel enrichment, average burnup at beginning of cycle $(13.4 \mathrm{GWd} / \mathrm{t}$ for cycle $\mathrm{A}$ and 14.8 for cycle B). Correlations tend to be larger between steps with similar burnups.

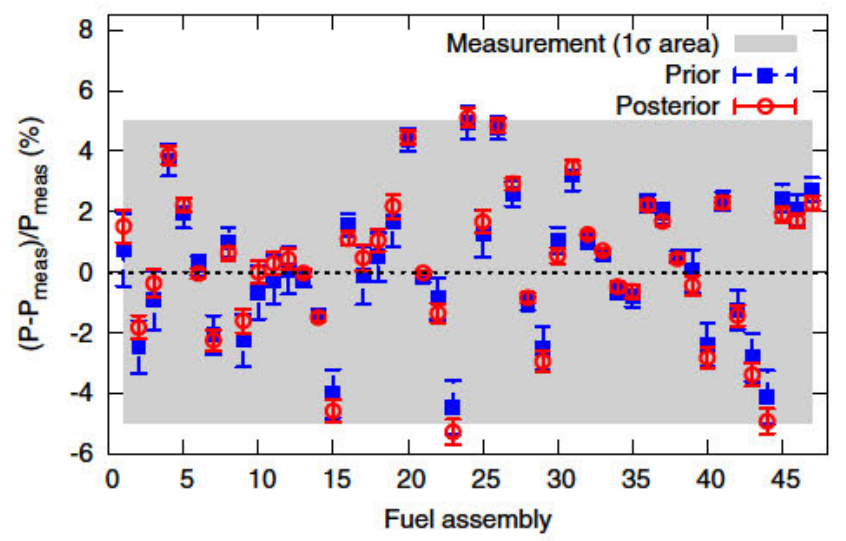

Fig. 9. Relative deviations of the prior and posterior powers per fuel assembly of Cycle B to its measurements at $2842 \mathrm{MWd} / \mathrm{t}$.
Fig. 9 compares the simulation results to the Cycle B measure ments. However, due to the low precision of the measurements, it cannot be judged whether the posterior or prior estimates give a better description. Both are consistent with the measurements.

The analysis shows that the uncertainty reduction for the power predictions by MOCABA is much less favorable than for the boron letdown curve predictions. This feature results from the high uncertainties in the power measurements of $5 \%$, which are far less precise than the boron measurements $(6 \mathrm{ppm} \equiv 0.6 \%$ uncertainty at BOC). The low precision of measurements can also not be bal anced by their large quantity ( 470 power measurements instead of 10 boron concentration measurements).

\subsection{Combining benchmarks and predictions of different responses}

With MOCABA, the prediction of a response can be improved by measurements of any benchmark observable. Thus it is also possi ble to update the power predictions by the boron concentration measurements and vice versa. In order to study this feature, we consider three scenarios for improving the power predictions:

- Updating based on power measurements (see previous section), - Updating based on boron concentration measurements,

- Updating based on all measurements.

Fig. 11 shows the results for a burnup of $2842 \mathrm{MWd} / \mathrm{t}$ during Cycle B. If the power distributions are updated only with boron concentration measurements, then the uncertainty is reduced in maximum by $33 \%$ and on average by $13 \%$. This rather modest improvement is explained by fairly low correlations of local power and boron concentration values. If the update considers both boron concentration and power measurements, then the uncertainty reduction amounts to on average $53 \%$ which is approx. $6 \%$ better than for considering only the power measurements. This demon strates that different responses can be combined in the Bayesian inference, and all of them can provide useful information.

\section{Prior (2842 MWd/t)}
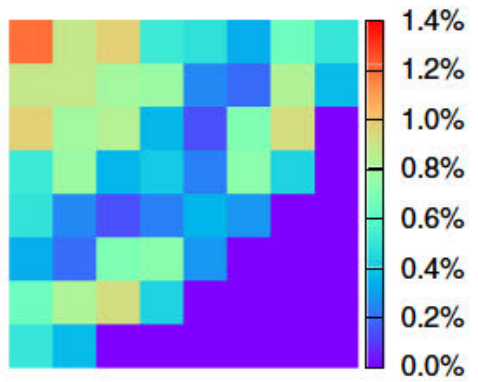

Prior (10284 MWd/t)

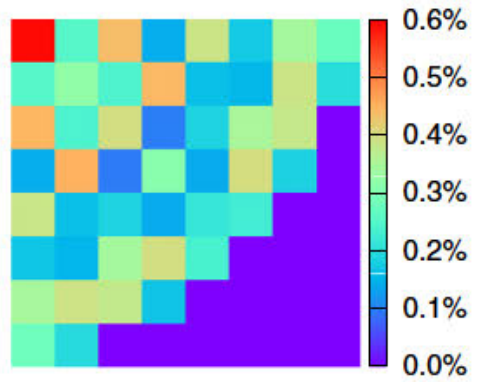

\section{Posterior (2842 MWd/t)}

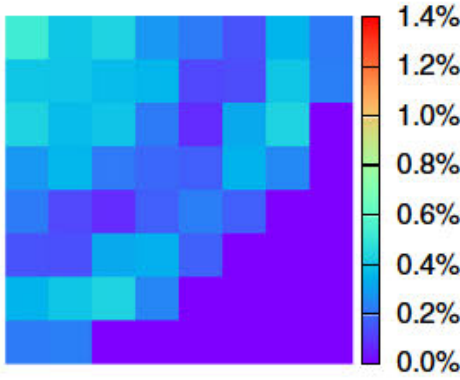

Posterior (10284 MWd/t)

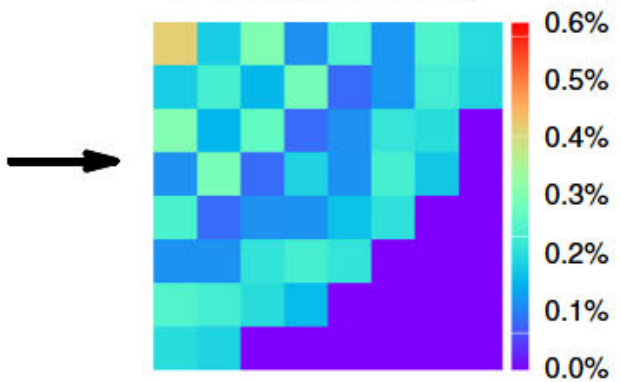

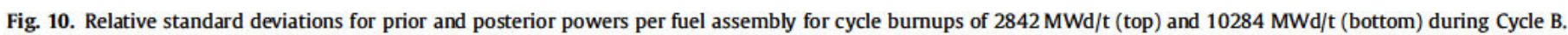




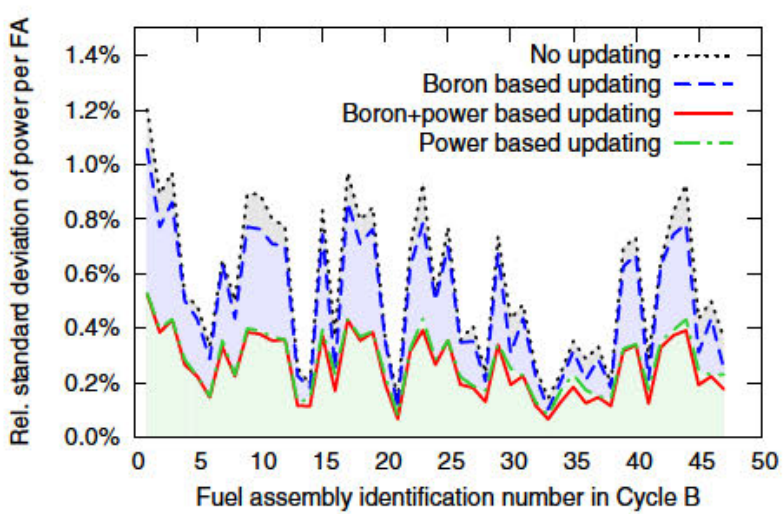

Fig. 11. Relative standard deviation, due to nuclear data uncertainties, of the power of each fuel assembly at the 47 locations in a quarter of the core, at $2842 \mathrm{MWd} / \mathrm{t}$. Updates performed using only power per fuel assembly measurements, only boron concentration measurements, and both.

\section{Conclusions}

The nuclear data Monte Carlo code NUDUNA has been applied to PWR core simulations with SEANAP to provide both best esti mates and their uncertainties for the boron letdown curve and the fuel assembly wise power distribution. Next, the predictions for a given reactor cycle (Cycle B) have been updated by applying the Bayesian inference model MOCABA, utilizing measurement information obtained in the preceding reactor cycle (Cycle A). The resulting updated best estimates and their uncertainties have been compared afterwards to the measurements during Cycle B in order to verify the predictive power of the procedure.

For the boron letdown curve, the MOCABA updating leads to major improvements for the best estimates and to massive uncer tainty reductions. The nuclear data induced uncertainty of 40 to $45 \mathrm{ppm}$ is reduced to $24 \mathrm{ppm}$ after applying MOCABA, i.e. to up to a 20 times lower uncertainty, caused by the low uncertainty in the measurements.

For the power per fuel assembly, the $5 \%$ relative uncertainty of the power measurements of Cycle A limits the possible reduction of the prediction uncertainty of Cycle B. Still, MOCABA provides a major uncertainty reduction of $53 \%$ on average. This analysis also demonstrates the ability of the MOCABA methodology to combine simulations and measurements of different response observables.

MOCABA has also been applied to generate an updated nuclear data library in WIMS format based on the boron concentration measurements of Cycle A. Computing the boron letdown curve for Cycle B with this updated library gives results that are almost identical to the ones obtained by direct MOCABA updating of the boron concentrations (differences are smaller than $2 \mathrm{ppm}$ ). The nuclear data in the resulting library lie within the one standard deviation range of the original data, and thus are compatible with them. However, the updating might also compensate for calcula tion code deficiencies or technological parameter uncertainties of the considered PWR plant. Future studies need to address the question whether the updated nuclear data obtained for one speci fic reactor with one specific reactor code suite can improve the description of other analyzes.

Predictions and measurements of the boron concentration and the power per fuel assembly appear to agree within their error bars even though system parameter uncertainties have not been taken into account in the analysis. This seems to support our assumption that the integral uncertainties are indeed governed by nuclear data uncertainties; see Section 2.4. It should, however, be noted that this assumption is not generally transferable to other application cases since it was linked to the expectation of strong error cance lation effects due to a high degree of similarity between bench mark and application case.

The blind tests presented in this paper show very good perfor mance of the NUDUNA/MOCABA best estimate plus uncertainty methodology. The obtained gain in precision, especially for the boron letdown curve, is impressive and promises major economi cal benefits. Compared to the traditional perturbative GLLS method, our Monte Carlo Bayes procedure has four major advan tages: it can be easily implemented since the transport codes used for the computation of the integral observables of interest can be treated as black boxes; it can easily address any integral function of nuclear data, such as boron concentration, power per fuel assembly, axial offset, and peak factors; integral observables can be directly updated without taking the detour via nuclear data updating, although also updated nuclear data libraries can be gen erated; and, due to its non perturbative nature, it is not limited to the regime where nuclear data uncertainties are small.

\section{Acknowledgements}

This work was supported by AREVA GmbH and conducted in the framework of the agreement in the area of Propagation of Uncertainties for Neutronic Calculations in Criticality Safety Anal ysis between the Spanish Nuclear Safety Council (CSN) and Univer sidad Politécnica de Madrid (UPM).

\section{References}

Ahnert, C., 1980. Programa WIMS-TRACA para el cálculo de elementos combustibles. Manual de usuario y datos de entrada, Madrid, Spain.

Ahnert, C, Aragonés, J.M., 1985. Fuel management and core design code systems for PWR neutronic calculations. Nucl. Technol. 69, 350-367.

Ahnert, C., Aragonés, J.M., Crespo, A., Labay, A., León, J.R., Alvarez, A.I., 1988. Validation of the pressurized water reactor core analysis system SEANAP-86 with measurements in tests and operation. Nucl. Sci. Eng. 100, 305-313.

Ahnert, C., Aragonés, J.M., Cabellos, O., Garća-Herranz, N., 1999. Continuous validation and development for extended applications of the SEANAP integrated 3-D PWR core analysis system. Math. Comput. Reactor Phys. Environ. Anal. Nucl. Appl. 1, 710-719.

Antila, M., Dusic, M., Fil, N., Glaeser, H., Hajra, P., Hortal, J., Mandowara, S., Misak, J., Nemes, I., Polyakov, A., Prosek, A., Rimkevicius, S., Stanev, I., Tkac, A. Victorov, A., Vymazal, P., Wellens, B., 2003. Safety Margins of Operating Reactors Tech. Rep. IAEA-TECDOC-1332. IAEA.

Aragonés, J.M., Ahnert, C., 1986. A linear discontinuos finite difference formulation for synthetic coarse-mesh few-group diffusion calculations. Nucl. Sci. Eng. 94 (4), 309-322.

Broadhead, B., Rearden, B., Hopper, C.M., Wagschal, J., Parks, C., 2004. Sensitivityand uncertainty-based criticality safety validation techniques. Nucl. Sci. Eng. $146,340-366$

Buss, O., Hoefer, A., Neuber, J., 2011. NUDUNA - nuclear data uncertainty analysis. In: Proc. International Conference on Nuclear Criticality (ICNC 2011), Edinburgh, Scotland.

Cabellos, O., Castro, E., Ahnert, C., Holgado, C., 2014. Propagation of nuclear data uncertainties for PWR core analysis. Nucl. Eng. Technol. 46 (3), 299-312.

Cecchini, G., Farinelli, U., Gandini, A., Salvatores, M., 1965. Analysis of integral data for few-group parameter evaluation of fast reactors. In: Proc. of the 3rd International Conference on Peaceful Uses of Atomic Energy, New York, USA.

Chadwick, M., Herman, M., Obložinský, P., Dunn, M., Danon, Y., Kahler, A., Smith, D. Pritychenko, B., Arbanas, G., Arcilla, R., Brewer, R., Brown, D., Capote, R., Carlson, A., Cho, Y., Derrien, H., Guber, K., Hale, G., Hoblit, S., Holloway, S., Johnson, T., Kawano, T., Kiedrowski, B., Kim, H., Kunieda, S., Larson, N., Leal, L., Lestone, J., Little, R., McCutchan, E., MacFarlane, R., Maclnnes, M., Mattoon, C, McKnight, R. Mughabghab, S., Nobre, G., Palmiotti, G., Palumbo, A., Pigni, M., Pronyaev, V., Sayer, R., Sonzogni, A., Summers, N., Talou, P., Thompson, L., Trkov, A., Vogt, R., van der Marck, S., Wallner, A., White, M., Wiarda, D., Young, P., 2011. ENDF/BVII.1 nuclear data for science and technology: cross sections, covariances, fission product yields and decay data. Nucl. Data Sheets 112 (12), 2887-2996, ISSN 0090375.

CSEWG, 2010. ENDF-6 Formats Manual. Data Formats and Procedures for the Evaluated Nuclear Data Files ENDF/B-VI and ENDF/B-VII, CSEWG Document ENDF-102 BNL-90365-2009 Rev. 1, Brookhaven National Laboratory, Upton, USA.

D’Auria, F., Glaeser, H., Lee, S., Mišák, J., Modro, M., Schultz, R., 2008. Best Estimate Safety Analysis for Nuclear Power Plants: Uncertainty Evaluation Tech. Rep. STI/ PUB/1306. LAEA 
De Saint Jean, C., Dupont, E., Ishikawa, M., Palmiotti, G., Salvatores, M., 2010. Assessment of Existing Nuclear Data Adjustment Methodologies, NEA/NSC/ WPEC/DOC(2010)429.

Díez, C.J., Buss, O., Hoefer, A., Porsch, D., Cabellos, O., 2015. Comparison of nuclear data uncertainty propagation methodologies for PWR burn-up simulations. Ann. Nucl. Energy 77, 101-114.

Gandini, A., 1967. A generalized perturbation method for bilinear functionals of the real and adjoint neutron fluxes. J. Nucl. Energy 21, 755.

Halsall, M.J., 1980. A Summary of WIMS-D4 Input Tech. Rep. AEEW-M-1327. U.K. Atomic Energy Establishment.

Hemment, P., Pendleburry, E., 1966. The optimization of neutron cross-section data adjustments to give agreement with experimental critical size. In: Proc. Int. Conf. on Fast Critical Experiments and Their Analysis, aNL, Illinois.

Hoefer, A., Buss, O., Hennebach, M., Schmid, M., Porsch, D., 2015. MOCABA: a general Monte Carlo - Bayes procedure for improved predictions of integral functions of nuclear data. Ann. Nucl. Energy 77, 514-521.

Humi, M., Wagschal, J., Yeivin, Y., 1965. Multi-group constants from integral data. In: Proc. of the 3rd International Conference on Peaceful Uses of Atomic Energy, New York, USA.

Ivanova, T., McKnight, R., Mennerdahl, D., Neuber, J., Rearden, B., Santamarina, A., Vasiliev, A., 2013. Overview of Approaches Used to Determine Calculational Bias in Criticality Safety Assessment: State-of-the-Art Report (Part 1), NEA/NSC/ WPNCS/DOC(2013) 7.

Ivanov, K., Avramova, M., Kamerow, S., Kodeli, I., Sartori, E., Ivanov, E., Cabellos, O., 2013. Benchmarks for uncertainty analysis in modelling (UAM) for the design, operation and safety analysis of LWRs, Volume I: Specification and Support Data for Neutronics Cases (Phase I), NEA/NSC/DOC(2013) 7.

Klein, M., Gallner, L., Krzykacz-Hausmann, B., Pautz, A., Velkov, K., Zwermann, W., 2012. Interaction of loading pattern and nuclear data uncertainties in reactor core calculations. In: Proc. Physics of Reactors International Conference (PHYSOR 2012), Knoxville, Tennessee, USA.

Koning, A., Rochman, D., 2008. Towards sustainable nuclear energy: putting nuclear physics to work. Ann. Nucl. Energy 35, 2024-2030.

Koning, A., Rochman, D., 2011. Modern nuclear data evaluation with the TALYS code system. Nucl. Data Sheets 113 (12), 2841-2934.

Leszczynski, F., Aldama, D.L., Trkov, A., 2007. WIMS-D Library Update: Final Report of a Coordinated Research Project Tech. Rep. STI/PUB/1264. IAEA
MacFarlane, R., Kahler, A.C., 2010. Methods for processing ENDF/B-VII with NJOY. Nucl. Data Sheets 111 (12), 2739-2890.

MacFarlane, R., Muir, D., Boicourt, R., Kahler, A., 2012. The NJOY Nuclear Data Processing System, Version 2012 Tech. Rep. LA-UR-12-27079. Los Alamos National Laboratory.

Oak Ridge National Laboratory, 2009. SCALE: A Modular Code System for Performing Standardized Computer Analysis for Licensing Evaluation, ORNL/ TM-2005/39, Version 6.0, Oak Ridge, Tennessee, USA.

Salvatores, M., Palmiotti, G., Aliberti, G., McKnight, R.. Archier, P., De Saint-Jean, C. Dupont, E., Herman, M., Shikawa, M., Su-gino, K., Ivanova, T., Ivanov, E., Kim, S.J., Kodeli, I., Trkov, A., Manturov, G., Pelloni, S., Perfetti, C., Rearden, B., Plompen, A., Rochman, D., Wang, W. Wu, H., Yang W., 2013. Methods and Issues for the Combined Use of Integral Experiments and Covariance Data. NEA/NSC/WPEC $\operatorname{DOC}(2013) 445$

Salvatores, M., Palmiotti, G., Cabellos, O., 2014. personal communication.

Sanchez, R., Mondot, J., Stankovski, Z., Cossic, A., Zmijarevic, I., 1988. APOLLO II: a user-oriented, portable, modular code for multigroup transport assembly calculations. Nucl. Sci. Eng. 100 (3), 352-362. http://dx.doi.org/10.13182 NSE88-3.

Sanz, J., Cabellos, O., Garcia-Herranz, N., 2008. ACAB-2008: ACtivation ABacus Code. NEA Data Bank. NEA-1839 ACAB.

Trkov, A., 2008. WILLIE Program, Operations on WIMS Cross Section Library Tech. Rep. IAEA-1408. IAEA.

Uchasev, L., 1964. Perturbation theory for the breeding ratio and other number ratios pertaining to various reactor processes. J. Nucl. Energy Part A/B 18, 571

Wieselquist, W, Zhu, T, Vasiliev, A, Ferroukhi, H., 2013, PSI methodologies for nuclear data uncertainty propagation with CASMO-5M and MCNPX: results for OECD/NEA UAM benchmark phase I. Sci. Technol. Nucl. Install.

Williams, M., Wiarda, D., Smith, H., Jessee, M., Rearden, B., Zwermann, W., Klein, M. Pautz, A. Krzykacz-Hausmann, B., Gallner, L., 2012. Development of a statistical sampling method for uncertainty analysis with SCALE. In: Proc. Physics of Reactors International Conference (PHYSOR 2012), Knoxville, Tennessee, USA.

X-5 Monte Carlo Team, 2003. MCNP - A General Monte Carlo N-Particle Transport Code, LA-CP-03-0245, Version 5, Los Alamos, New Mexico, USA.

Zwermann, W., Krzykacz-Hausmann, B., Gallner, L., Pautz, A., 2010. Uncertainty analyses with nuclear covariance data in reactor core calculations. In: Proc. Jahrestagung Kerntechnik, Berlin, Germany. 